\title{
Description and Performance of a Highly Sensitive Confocal Raman Microspectrometer
}

\author{
G. J. Puppels, * W. Colier, J. H. F. Olminkhof, C. Otto, F. F. M. de Mul and J. Greve \\ Biophysical Technology Group, Department of Applied Physics, University of Twente, P.O. Box 217, 7500 AE Enschede, \\ The Netherlands
}

\begin{abstract}
A confocal Raman microspectrometer was developed for the study of small biological objects such as single living cells and metaphase and polytene chromosomes. It employs a confocal detection scheme, well known from confocal fluorescence microscopes, in order to avoid signal contributions from the environment of the samples. The resolution is $0.45 \pm 0.05 \mu \mathrm{m}$ in the lateral direction and $1.3 \pm 0.1 \mu \mathrm{m}$ in the axial direction. The laser excitation wavelength is $660 \mathrm{~nm}$. At this wavelength biological samples do not degrade in the laser radiation as was the case when laser radiation of $514.5 \mathrm{~nm}$ was used. The signal throughput from the sample position to the detector was optimized to the extent that in the spectral region around a $1000 \mathrm{~cm}^{-1}$ Raman shift $15 \%$ of the Raman scattered light collected by the microscope objective is detected. For signal detection a liquid nitrogen-cooled slow-scan CCD camera is used. Laser powers of $\mathbf{5 - 1 0} \mathbf{~ m W}$ suffice to obtain high-quality Raman spectra, with signal integration times of the order of minutes. As an example, spectra obtained from the nucleus and the cytoplasm of an intact human lymphocyte are shown.
\end{abstract}

\section{INTRODUCTION}

Raman spectroscopy is a valuable technique for the study of biological (macro)molecules, giving information about overall conformation and composition of these molecules and about the chemical microenvironment of constituents. $^{t}$ Because of the low Raman scattering cross-sections of nucleic acids, proteins and lipids, until recently most studies had to be performed on model systems. The investigation and characterization of these molecules as present in single living cells and intact subcellular structures was not possible. An exception was the study of the conformation of the highly compacted DNA in a single salmon sperm head. ${ }^{2}$ Recently, resonance Raman spectroscopy has been applied successfully to obtain a DNA spectrum from the nucleus of a human mammary tumour cell. ${ }^{3}$ Resonance Raman spectroscopy has also been used for the identification of single bacterial cells. ${ }^{4}$ We report here the development and performance with respect to sensitivity and spatial resolution of a confocal Raman microspectrometer (CRM) that has enabled us to record high quality nonresonant Raman spectra of single living cells and chromosomes. 5

\section{EXPERIMENTAL}

A Spectra Physics Model 375 B dye laser operated with DCM and pumped by an argon-ion laser (Coherent Radiation Innova 90) was used in the Raman experiments. It is equipped with a double birefringent plate and an ultrafine-tuning étalon to produce a line width of about $0.3 \mathrm{~cm}^{-1}$.

\footnotetext{
* Author to whom correspondence should be addressed.
}

The CRM (see Fig. 1) was developed in our laboratory. All spherical lenses in the CRM are achromats (except $\left.\mathbf{L}_{4}\right)$ and are anti-reflection coated $(R<1 \%$ per surface). Band-pass filters, silver mirrors and antireffection coatings were produced in the thin-film laboratory of our department.

Transmission curves of band-pass filters were recorded on a Beckman DU-8 spectrophotometer and corrected for reflection losses at the rear surface of the filters.

\section{Sample preparation}

Polystyrene beads used in the experiments were obtained from Polysciences (Warrington, PA, USA). A drop of diluted bead suspension was placed on a poly-L-lysine (Sigma, St. Louis, MO, USA) coated fused-silica substrate, and placed in a $100 \%$ humidity environment for $30 \mathrm{~min}$, then the substrate was rinsed with water. In this way a substrate with firmly attached beads was obtained.

Glass substrates with a $100-$ nm polystyrene coating were produced by spinning. The thickness of the polystyrene layer was measured with a Sloan Dektak 3030 stylus profiler.

\section{Determination of the CRM measuring volume}

Polystyrene beads of $0.22 \mu \mathrm{m}$ diameter were moved through the focused laser beam. The Raman signal was recorded as a function of the position of the bead relative to the centre of the laser focus.

The step resolution of the microscope scan table (Marzhauser EK-32) was $0.1 \mu \mathrm{m}(X, Y$, orthogonal and parallel to the polarization of the laser light). Vertical displacement $(Z)$ of the microscope table was achieved by a stepper motor connected to the microscope frame. $Z$-displacement was read at the microscope vertical 
positioning knob, which was calibrated. Step increments of $0.25 \mu \mathrm{m}$ were used in $Z$-scan measurements. This scanning option (being further developed at present) was used to make linear scans in the $X, Y$ and $Z$ directions. All measurements were preceded by positioning ( $Z$-steps $<0.1 \mu \mathrm{m}$ ) of a bead in the laser focus. The best position was where a maximum in the Raman signal from the bead was obtained. From this position the polystyrene bead was moved to the starting position of a scan. In this way $X, Y$ and $Z$ scans were made through the centre of the laser focus in order to determine lateral and axial resolution. $Z$-Scans were made moving the bead in positive and negative $Z$-directions through the laser focus, in order to ensure that the asymmetry in the depth response curve (Fig. 7) was not due to hysteresis in the stepper motor. Figures 7, 9(a) and 9 (b) are the results of averaging five scans, each on a different bead. This was done by projecting the response curves obtained from single scans in one graph in such a way that there was maximum overlap. The uncertainty in the full width at half maximum intensity (FWHM) value of the averaged curves refers to the maximum spread in the experimental results. For the purpose of obtaining a more complete impression of the three-dimensional form of the measuring volume, these measurements were complemented by $Z$-scans with the bead positioned at distances of $0.1,0.2$ and $0.3 \mu \mathrm{m}$ from the optical axis. The same averaging (in this case over three scans) of results as described above was applied. Off-optical-axis scans were only made with displacements from the optical axis along the positive $Y$-axis, because the scans along the $X$-and $Y$-axes mentioned above did not show asymmetry. For the reconstruction of the measuring volume in three dimensions a cylindrical symmetry around the optical axis was therefore assumed.

A computer program was written to simulate the experiments in order to estimate the broadening of the response curve due to the finite dimensions of the polystyrene bead. In this simulation the intensity profile of the laser radiation in the focal plane was assumed to be a sinc ${ }^{2}$ function. The polystyrene bead was modelled as a mass $(M)$ distribution $\left[M(r) \sim \sqrt{1-r^{2} / r_{\text {bead }}}\right]$ in the focal plane. In this way the calculations were reduced to two dimensions (in the focal plane). Effects due to reflection, refraction and diffraction were neglected.

The lateral optical unit (lou), used in the section on confocal properties, is defined as lou $=(n k \sin x)^{-1}$ (where $n \sin \alpha$ is the numerical aperture of the objective and $k=2 \pi(\lambda)$. The radius of the pinhole in lou refers to the radius as projected in the object plane of the microscope objective.

\section{Determination of the relative detection efficiency of the CRM}

The $2215 \pm 15 \mathrm{~K}$ spectrum of a tungsten band lamp (Philips $6002 \mathrm{E}$ ) positioned under the microscope objective was measured. The black-body temperature of the tungsten band was measured with a pyrometer (Hartmann and Braun). This value was corrected by taking into account the tungsten emission coefficient to obtain the actual temperature of the tungsten band. The spectrum of the light emitted by the lamp was calcu- lated from Wien's law. Hence the relative amount of light incident on each pixel of the CCD detector in the case of wavenumber-independent CRM signal transmission could be determined. Normalizing the ratio of the detected and calculated amounts of light at one point in the spectrum, the relative detection efficiency of the CRM was determined for the Stokes Raman shift spectral interval of $0-3500 \mathrm{~cm}^{-1}$.

To determine the relative detection efficiency for the two polarization directions (parallel and orthogonal to the polarization of the laser radiation) separately, a Spindler and Hoyer Type $10 \mathrm{~K}$ polarization filter was installed in the set-up and the measurements were repeated for both polarizations. The polarizing ratio of the filter is wavelength dependent and decreases at higher wavelengths. At $790 \mathrm{~nm}\left(2500 \mathrm{~cm}^{-1}\right.$ Stokes Raman shift) it was still found to be $>50: 1$. For wavelengths $>790 \mathrm{~nm}$ an extra filter was installed so that throughout the whole spectral interval $\left(0-3500 \mathrm{~cm}^{-1}\right.$ Stokes Raman shift) a minimum polarization ratio of $>50: 1$ was maintained, sufficient for these measurements.

Also, (polarized) light transmission of the filter(s) is wavelength dependent. The detection efficiency measurements were corrected for this effect. The detection efficiency curves for the two polarizations were scaled by comparing the absolute number of counts detected for both polarizations at a number of positions in the band lamp spectrum. The light from the band lamp was found to be slightly polarized. A difference in intensity of about $5 \%$ between the two polarization directions was observed. Therefore, these measurements were carried out in duplicate with the lamp placed under the microscope objective in two mutually orthogonal positions. The results were averaged. The curve for unpolarized light was then scaled in such a way that it coincided with the points of equal detection efficiency for the two polarized light curves.

\section{Lymphocyte sample preparation}

The mononuclear white blood cell fraction was isolated from human peripheral blood, obtained by venipuncture, according to the method described previously. ${ }^{6}$ To remove monocytes from this fraction, the cells were incubated in a $75-\mathrm{cm}^{2}$ tissue culture flask for $1 \mathrm{~h}$ at $37^{\circ} \mathrm{C}$. This caused the monocytes to adhere to the bottom surface of the flask, whereas lymphocytes remained in suspension. A drop of this suspension was then placed on a poly-L-lysine-coated fused-silica substrate placed in a Petri dish. After a few minutes of incubation in a $100 \%$ humidity environment, the cells were attached to the substrate. The Petri dish was then filled with Hank's balanced salt solution (HBSS, according to Gibco 041-04025 without phenol red).

\section{RESULTS}

By means of Raman microspectroscopy, spectral information can be obtained from specific (small) regions in a sample. ${ }^{7.8}$ Our aim in developing the CRM was to be able to record Raman spectra of small volumes $(\leqslant 1$ $\mu^{3}$ ) of biological material with spatially resolved mea- 
surements in cells and chromosomes. This implies that the amount of DNA is of the order of only $10-100 \mathrm{fg}$ in the volume observed. Of course, the laser power on the sample should not exceed a level above which it would cause degradation or destruction of the sample. Achieving the required sensitivity was therefore the principal design criterion. The CRM was optimized for efficient signal collection combined with good suppression of the background signal (from buffer and substrate), high throughput of the Raman signal through the spectrograph and multi-channel signal detection with a lownoise, high quantum efficiency detector, free from dark current.

\section{Collection, throughput and detection of Raman signal}

The optical configuration of the CRM is shown in Fig. 1. Laser radiation at $660 \mathrm{~nm}$ from a DCM-operated dye laser is focused on a sample by means of a high-power microscope objective after passing through a narrow band-pass filter (BPF). The BPF has a transmission of $84 \%$ for the laser radiation. The width of the light cone entering the objective is such that $30 \%$ of the laser radiation is lost by apodization. This ensures utilization of the full numerical aperture of the objective. As will be shown below, the FWHM of the intensity in the laser focus is $<0.5 \mu \mathrm{m}$. Scattered and reflected light are collected by the same objective. The BPF reflects the Raman scattered light (reflection coefficient $>98 \%$ in the spectral interval $300-3000 \mathrm{~cm}^{-1}$ ). This light is focused in the image plane of the microscope objective, where a pinhole is positioned. The pinhole serves to limit the measuring volume (described in the section on confocal properties). The light passing through the pinhole is collected by a positive lens which forms a parallel light beam, necessary to use the Chevron-type band-pass filter set. ${ }^{9}$ This filter set consists of two parallel-mounted narrow band-pass filters. Light is reflected back and forth between these filters. At each reflection $>80 \%$ of the laser light is transmitted and thus effectively separated from the Raman scattered light, which is very efficiently reflected (reflection coefficient $>99 \%$ between 600 and $2600 \mathrm{~cm}^{-1}$ Stokes Raman shift). Using 12 reflections, a laser line suppression of $10^{8}-10^{9}$ is obtained.

The throughput for the Raman signal is shown in Fig. 2. After expanding the light beam $\left(L_{3}\right.$ and $\left.L_{4}\right)$, a ruled grating of 300 lines $\mathrm{mm}^{-1}$ and a $600-\mathrm{nm}$ blaze (Jobin Yvon) is used for wavelength dispersion. A concave mirror of $0.45-\mathrm{m}$ focal length focuses the Raman spectrum on the liquid nitrogen-cooled slow-

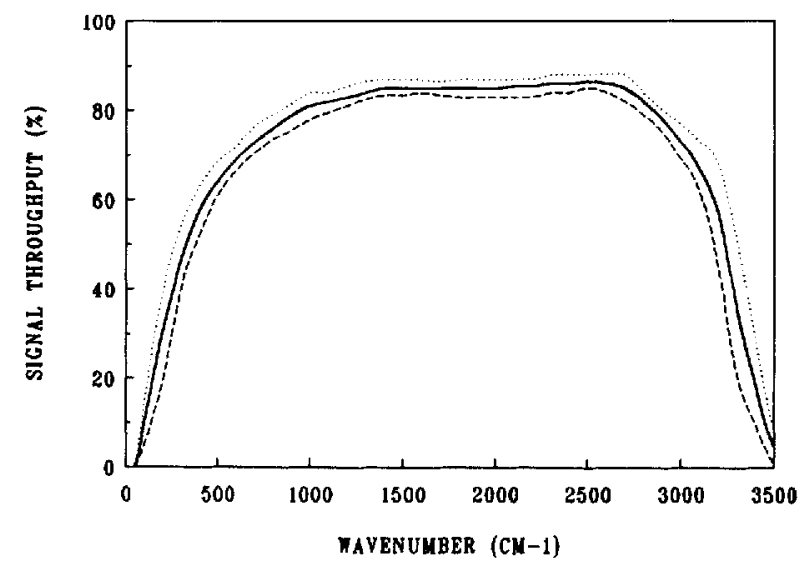

Figure 2. Throughput for Raman signal of the Chevron-type band-pass filter set. Solid line, unpolarized; dotted line, parallel laser polarization; dashed line, perpendicular laser polarization; see Fig. 1, detailed description in Ref. 9.

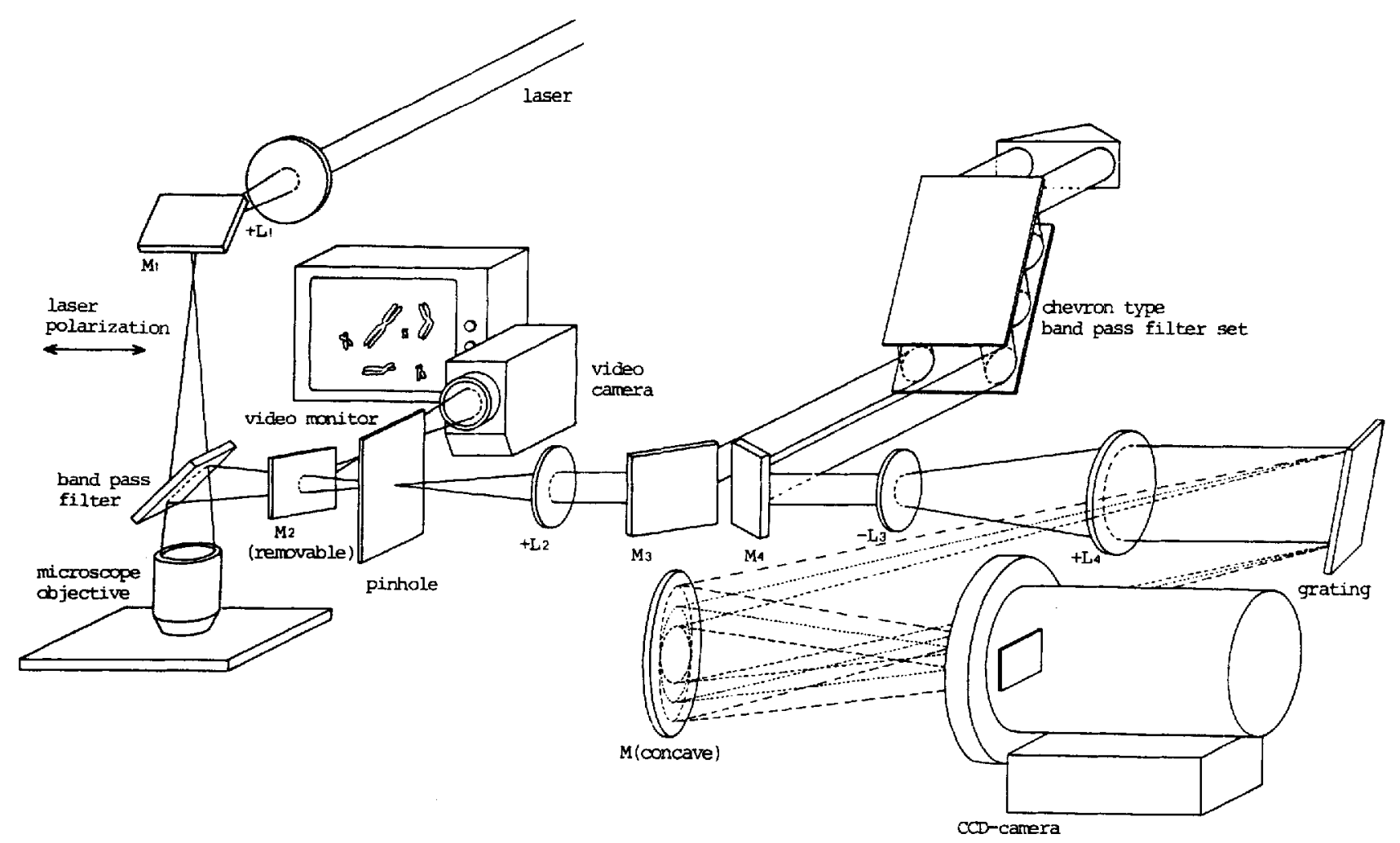

Figure 1. The confocal Raman microspectrometer (CRM). 
scan CCD camera (Wright Instruments, EEV P 8603 CCD chip). The most important spectral interval (600$1750 \mathrm{~cm}^{-1}$ Stokes Raman shift), for our applications, can thus be covered in a single measurement. In the direction of wavelength dispersion only 385 camera pixels are available. This means that on average 1 pixel corresponds to $3 \mathrm{~cm}^{-1}$. In the case of narrow Raman lines this may give rise to aliasing effects, which degrade the spectral resolution. This was considered to be of minor importance for the present applications, however. The recorded spectra are routinely interpolated from the 385 measured points to 1000 points in order to linearize the wavenumber scale and for software compatibility in further processing of the data. The measured laser line width (after interpolation) was found to be 6-7 $\mathrm{cm}^{-1}$. Apart from aliasing effects, the spectral resolution is therefore $6-7 \mathrm{~cm}^{-1}$.

The quantum efficiency of the CCD camera has a peak value of about $40 \%$ around $700 \mathrm{~nm}$. It is cooled to $140 \mathrm{~K}$. This eliminates dark current. With the double correlated sampling method, employed in the camera electronics, reset noise is avoided. ${ }^{10}$ Only 10 electrons of read-out noise, per measuring point, remain (equalling 10 detected photons). The signal-to-noise ratio of the measurements is therefore photon noise limited at virtually any signal level. The detection of cosmic ray events (CREs) leads to spikes in the recorded spectrum which on average equal 2000 detected photons. To minimize the chance of CRE detection, as few pixels as possible should be used in the direction orthogonal to dispersion. A cylindrical lens in front of the CCD camera (not shown in Fig. 1) is employed to correct for the astigmatism due to the off-optical-axis focusing by the concave mirror. In this way all the Raman signal is contained in 10 pixels (orthogonal to dispersion, with $90 \%$ of the signal confined to 5 pixels). As only these (binned) pixels are read out, CREs are rarely detected. Pixel-to-pixel sensitivity variations are routinely removed from the Raman spectra by a method similar to that described by Howard and Maynard. ${ }^{11}$

Figure 3 shows the overall detection efficiency for the Raman signal of the CRM (polarized and unpolarized) as a function of wavenumber shift. A calculation of the absolute detection efficiency, partly (grating, CCD camera) based on suppliers' data sheets, shows that of all the light collected by the microscope objective up to

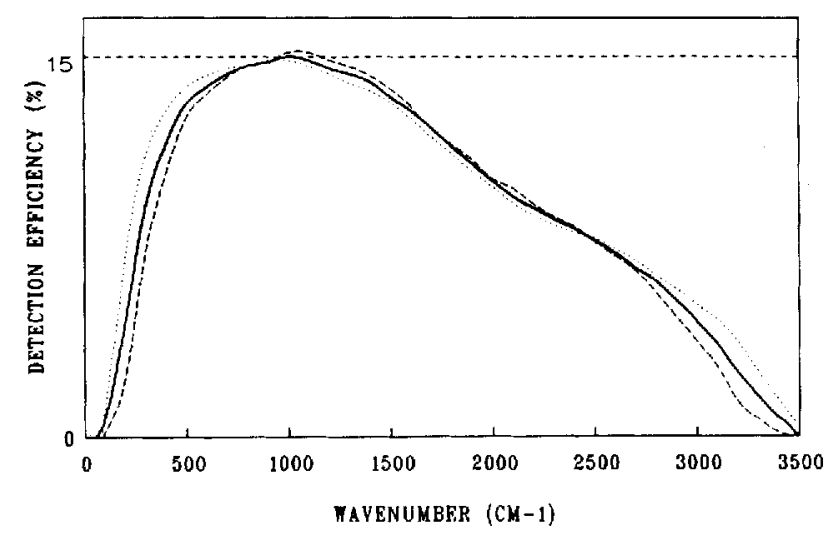

Figure 3. Raman signal detection efficiency of the CRM (percentage of photons detected after collection by the microscope objective). Lines as in Fig. 2. about $15 \%$ is actually detected (Table 1). In a large part of the spectrum the detection efficiency is almost polarization independent. This permits easy correction of recorded spectra for the wavenumber dependence of the detection efficiency, without the need for either measuring the two polarization components of the Raman scattered light separately or using a polarization scrambler at the entrance of the spectrometer. The same method used for obtaining the detection efficiency curve in Fig. 3 can be used for this purpose (see Experimental).

\section{Confocal properties, measuring volume}

Many papers have been published about the principles and applications of confocal microscopy. ${ }^{12-19}$ Especially in the form of confocal scanning laser fluorescence microscopy it has readily found application in cellular biology. Its greatest merit is the suppression of fluorescence from out-of-focus regions of the object under study, which otherwise tends to blur images. This 'optical sectioning' capability allows the reconstruction of objects in three dimensions. Figure 4 shows the basic optical configuration of a confocal microscope. It makes

\section{Table 1. Calculation of the absolute detection efficiency of the CRM (at $1000 \mathrm{~cm}^{-1}$ shift)}

$\begin{array}{lc}\text { Component } & \begin{array}{c}\text { Throughput }(\%) \\ \text { at } 1000 \mathrm{~cm}^{-1} \text { shift }\end{array} \\ \text { Objective } & 85^{\circ} \\ \text { BPF } & 98 \\ \text { Lenses and concave mirror } & 90 \\ \text { Chevron-type band-pass } & 80 \\ \quad \text { filter set (including mirrors) } & \\ \text { Grating } & 68^{\circ} \\ \text { CCD camera } & 40^{\circ} \\ \text { Total } & \sim 15 \% \\ \text { a Measured at } 660 \mathrm{~nm} . & \\ \text { 'Jobin Yvon data sheet, corrected for Al reflec- } \\ \text { tance. } \\ \text { "Ref. } 10 .\end{array}$

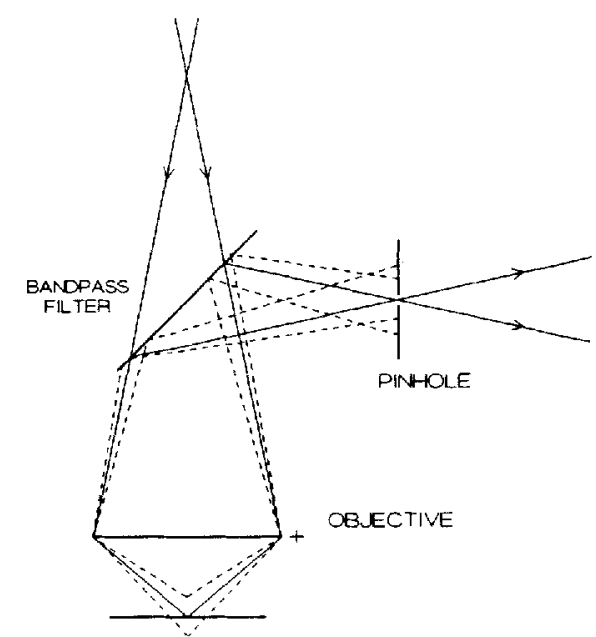

Figure 4. Suppression of out-of-focus signal contributions in the confocal microscope. 
clear how an out-of-focus signal is blocked by the pinhole in the image plane of the microscope objective. The depth or axial resolution that is achieved in this way depends on the pinhole radius. Using high numerical aperture objectives and a pinhole of a radius less than $2.5 \mathrm{lou}$, an axial resolution of $0.7 \mu \mathrm{m}$ (FWHM) can be obtained. ${ }^{16,17.19}$ If the pinhole radius is reduced to less than $0.5 \mathrm{lou}$, the lateral optical resolution can be improved by a factor of $1.4,{ }^{12,17}$ compared with the classical diffraction limit. However, the accompanying loss in signal intensity often prohibits the use of such small pinholes.

The problem of very low signal intensities when considering Raman spectroscopy of small biological samples such as a metaphase chromosome is aggravated by strong contributions to the Raman signal from the environment of the sample (buffer, substrate). These background contributions, which have to be subtracted in order to obtain the desired spectrum, lead to a decrease in the signal-to-noise ratio. This problem can be compared with the out-of-focus blur in fluorescence microscopy. In principle it can be avoided in the same way as was done in confocal fluorescence microscopes (CFMs). This was described by Etz and Blaha for their Raman microprobe. ${ }^{20}$ Because of the inherently low signal intensity in Raman spectroscopy, optimizing the pinhole size is even more important in a CRM than in a CFM. Loss of signal from the object under investigation should be avoided.

In the CRM a $63 \times$ Zeiss water immersion objective (NA 1.2) is used routinely. Figure 5 shows how the signal intensity depends on pinhole size. The intensity of the $1004 \mathrm{~cm}^{-1}$ Raman band of a $0.5-\mu \mathrm{m}$ polystyrene bead on a fused-silica substrate in water and the intensity of the background signal between 400 and 500 $\mathrm{cm}^{-1}$ were measured for three different pinhole sizes and also without a pinhole installed [Fig. 5(a)]. It is clear that using a pinhole with a radius of $50 \mu \mathrm{m}$ (9 lou) a considerable suppression of the background signal is obtained, without a significant loss of signal from the polystyrene bead. Figure 5(b) shows the Raman spectra. In our applications (single cells and chromosomes), the background signal is usually between 5 and 20 times stronger than the signal from the sample without the use of a pinhole. The best signal-to-noise ratio is then obtained by using the $100-\mu \mathrm{m}$ pinhole. It leads to an improvement of the SN-ratio by a factor 2-2.4 or a reduction in measuring time by a factor 4-6 in comparison with the situation where no pinhole is used. (Other pinhole radii between 25 and $50 \mu \mathrm{m}$ have not been tested.)

The use of a pinhole of this size, however, leads to a different stituation than is found in a standard CFM. Experiments were carried out to analyse the confocal properties of the CRM, and to determine the measuring volume, from which the signal is collected.

The confocal behaviour of the CRM is brought about by two effects that can be analysed separately. If an object is moved away from the laser focus along the optical axis, light intensity will decrease as the distance to the focus increases. Consequently, the Raman signal from this object will also decrease if the dimensions of the object are smaller than that of the light cone [Fig. 6(a)]. As will be shown, depth resolution for point-like objects is mainly determined by this confocal effect
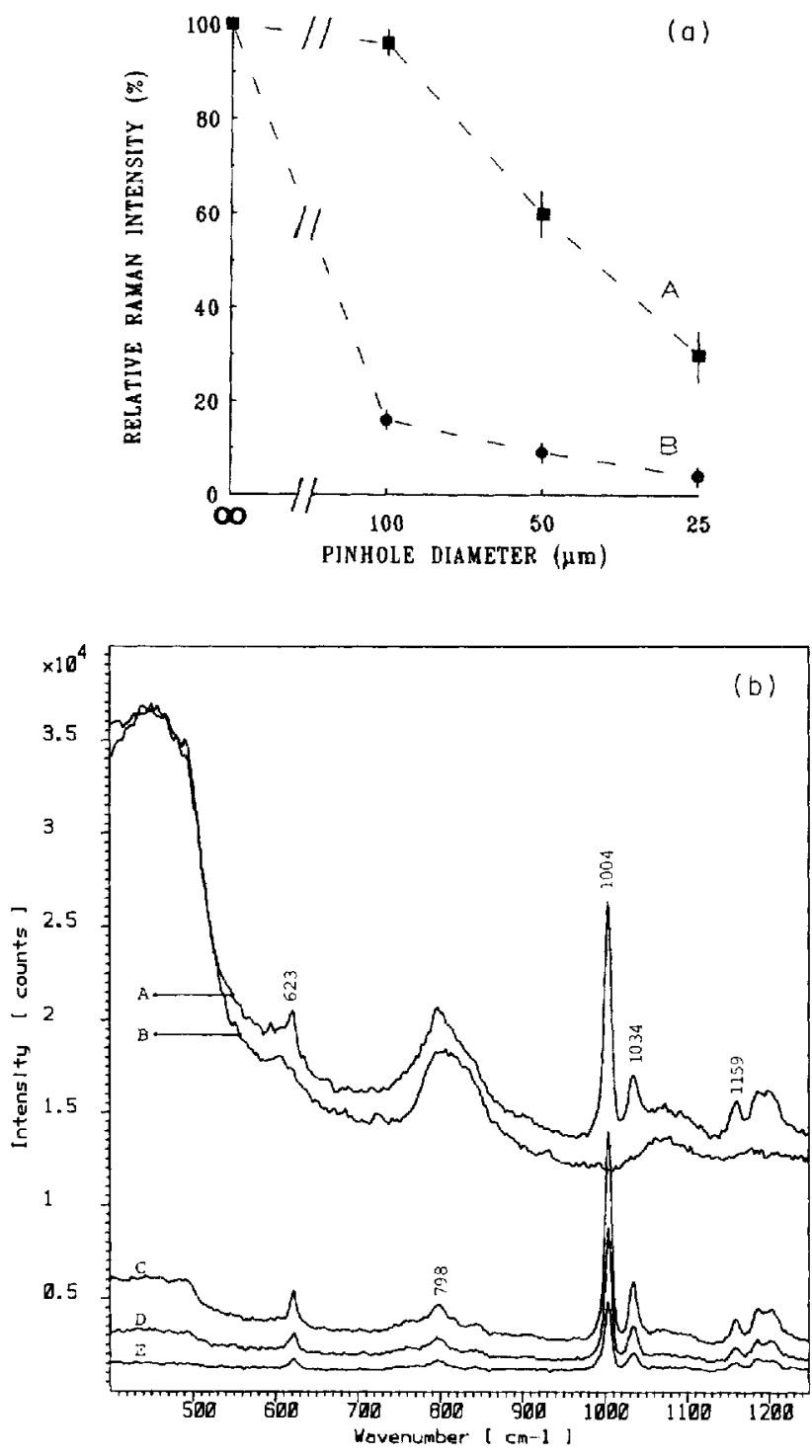

Figure 5. Influence of the size of the pinhole on signal ( $\square$ ) and background signal (O) intensity as measured for a $0.5-\mu \mathrm{m}$ polystyrene bead on a fused-silica substrate in water. (a) Graph showing decrease in (A) polystyrene signal and (B) background signal intensity. (b) Corresponding Raman spectra: (A) without pinhole; (B) background without pinhole; (C) with pinhole of $50-\mu \mathrm{m}$ (9-lou) radius; (D) with pinhole of $25-\mu \mathrm{m}$ (4.5-lou) radius: (E) with pinhole of $12.5-\mu \mathrm{m}(2.25$-lou) radius. Experimental conditions: laser power, $15 \mathrm{~mW}(660 \mathrm{~nm})$; microscope objective, $63 \times$ Zeiss Plan Neofluar water immersion (1.2 NA); signal integration time, $10 \mathrm{~s}$.

(CFE1) (a similar effect has been described ${ }^{21}$ for a fluorescence microscope). The second confocal effect is the result of the pinhole in the image plane of the microscope objective (Fig. 4, CFE2). Depth resolution for planar $(X Y)$ objects will be shown to depend mainly on this effect. (The environment of a sample, which causes the background signal, can be imagined as a multilayer of $X Y$ planes.)

To determine separately the influence of both effects on the point response of the CRM in an axial direction, the following experiments were carried out. The Raman signal of a small $(0.22-\mu \mathrm{m}$ diameter) polystyrene bead was measured as a function of distance to the laser focus, without a pinhole in place. In this way the depth response due to CFE1 was determined. The result, normalized with respect to the highest signal intensity, is 
(a)

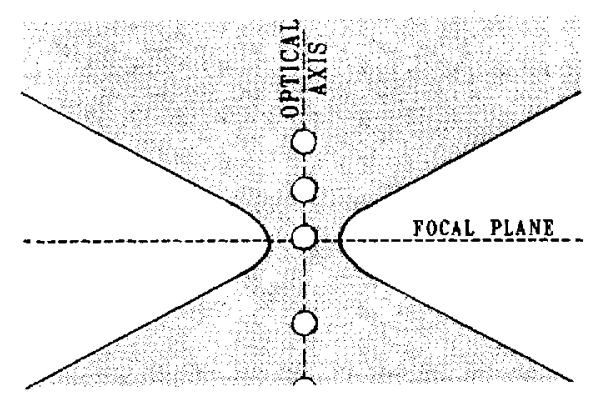

(b)

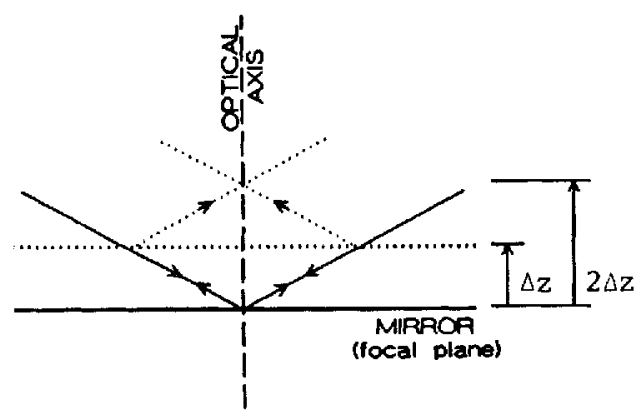

Figure 6. (a) CFE1 : a decrease in laser light intensity on the point object leads to a confocal effect. Shaded area indicates laser light cone. (b) CFE2: a constant intensity point-like light source is mimicked. The pinhole in the image plane of the objective determines the depth response.

shown in Fig. 7(B). The depth response due to CFE2 was determined by measuring the intensity of laser radiation that passes through the pinhole, after being reflected by a mirror that was moved through the laser focus. In that way a light source of constant intensity moving along the optical axis at twice the rate of the mirror was mimicked [see Fig. 6(b)]. This resulted in curve A in Fig. 7. It shows clearly that CFE 1 is the dominant effect. Curves $\mathrm{C}$ and $\mathrm{D}$ show that the combined action

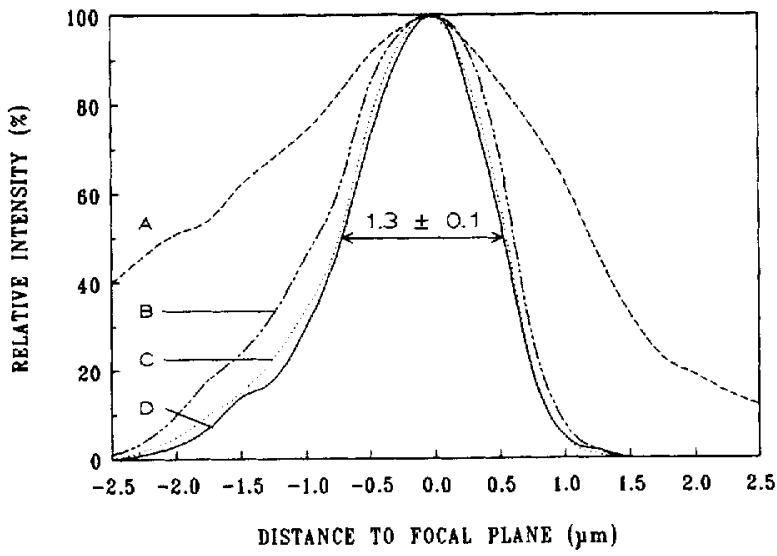

Figure 7. Depth response of the CRM for point-like objects. A, B and $D$ are averaged over five scans. The error in the FWHM values is based on the spread in these values found in different scans. (A) Response curve due to CFE2 (FWHM $=3.3 \pm 0.3$ ), pinhole radius $=50 \mu \mathrm{m}$. (B) Response curve due to CFE1 (FWHM $=1.5 \pm 0.1$ ). (C) Calculated combined action of CFE1 and CFE2 (multiplication of curves $A$ and $B$; FWHM = 1.3). (D) Experimentally determined combined action of CFE1 and CFE2 ( $F W H M=1.3 \pm 0.1$ ), pinhole radius $=50 \mu \mathrm{m}$. of the two confocal effects is found by multiplying curves $A$ and $B$. Curve $C$ is the result of multiplication of curves $A$ and $B$. Curve $D$ is the result of recording the Raman signal of the small polystyrene bead as a function of distance to the laser focus as for curve A, but with the pinhole installed. CFE2 becomes more important when smaller pinholes are used, and dominant for the 12.5- $\mu \mathrm{m}$ (2.25-lou) and smaller pinholes (not shown), as in the case of confocal fluorescence microscopy.

For the suppression of the background signal it is important to look at the depth response for planes. When a planar object is moved through the laser focus along the optical axis, the light intensity on the object decreases as for a point object, but since the product of intensity and irradiated surface remains constant, there will be no decrease in Raman signal intensity from this object. This is shown in Fig. 8(A). A glass substrate covered with a thin layer $(100 \mathrm{~nm})$ of polystyrene was moved through the laser focus. The intensity of the 1004 $\mathrm{cm}^{-1}$ Raman band was measured as a function of distance to the laser focus, without a pinhole in place. (The signal decrease at distances $>1 \mu \mathrm{m}$ from the focal plane is caused by apodization in the spectrometer.) The experiment was repeated after installing the $50-\mu \mathrm{m}$ pinhole [Fig. 8(B)]. It is clear that the depth response for planar objects depends mainly on CFE2.

The dimensions and form of the measuring volume from which the Raman signal is obtained were determined by scanning the region around the laser focus with a small polystyrene bead $(0.22 \mu \mathrm{m}$ diameter), as described under Experimental. The bead was moved through the laser focus in the two lateral directions [Figs. 9(a) and (b)], and the intensity of the $1004 \mathrm{~cm}^{-1}$ Raman band was again recorded as a function of the position of the bead with respect to the centre of the laser focus. For both lateral directions (parallel and orthogonal to the polarization direction of the laser radiation) the FWHM of the resulting curves is $0.45 \pm 0.05 \mu \mathrm{m}$. A computer simulation of the experiment was made in which the laser radiation intensity profile in the focal plane was modelled as a sinc ${ }^{2}$ function. The simulated and measured response curves coincide well until the $30 \%$ intensity level. At that point the

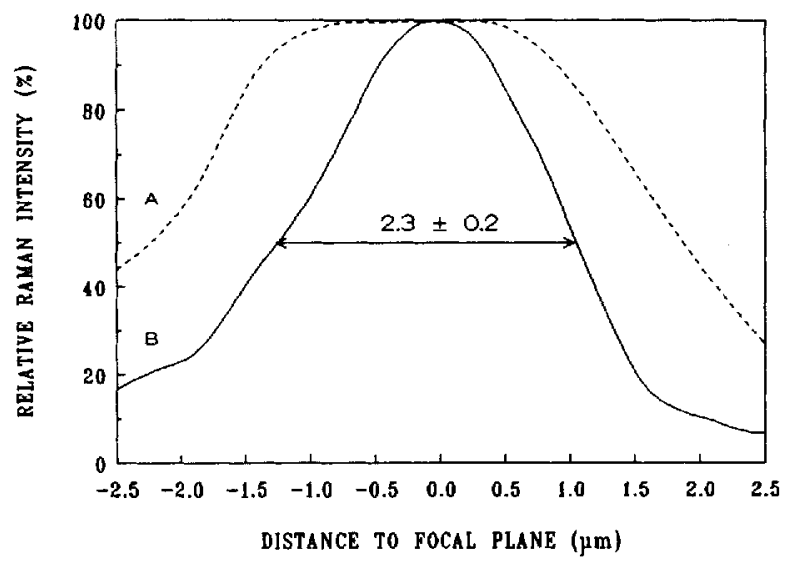

Figure 8. Depth response of the CRM for $(X Y)$ planar objects [averages of (A) three scans and (B) five scans]. (A) Response curve without a pinhole in place (FWHM $=4.2 \pm 0.3$ ). (B) Response curve with a pinhole (radius $50 \mu \mathrm{m}$ ) in place (CFE2, FWHM $=2.3 \pm 0.2$ ). 

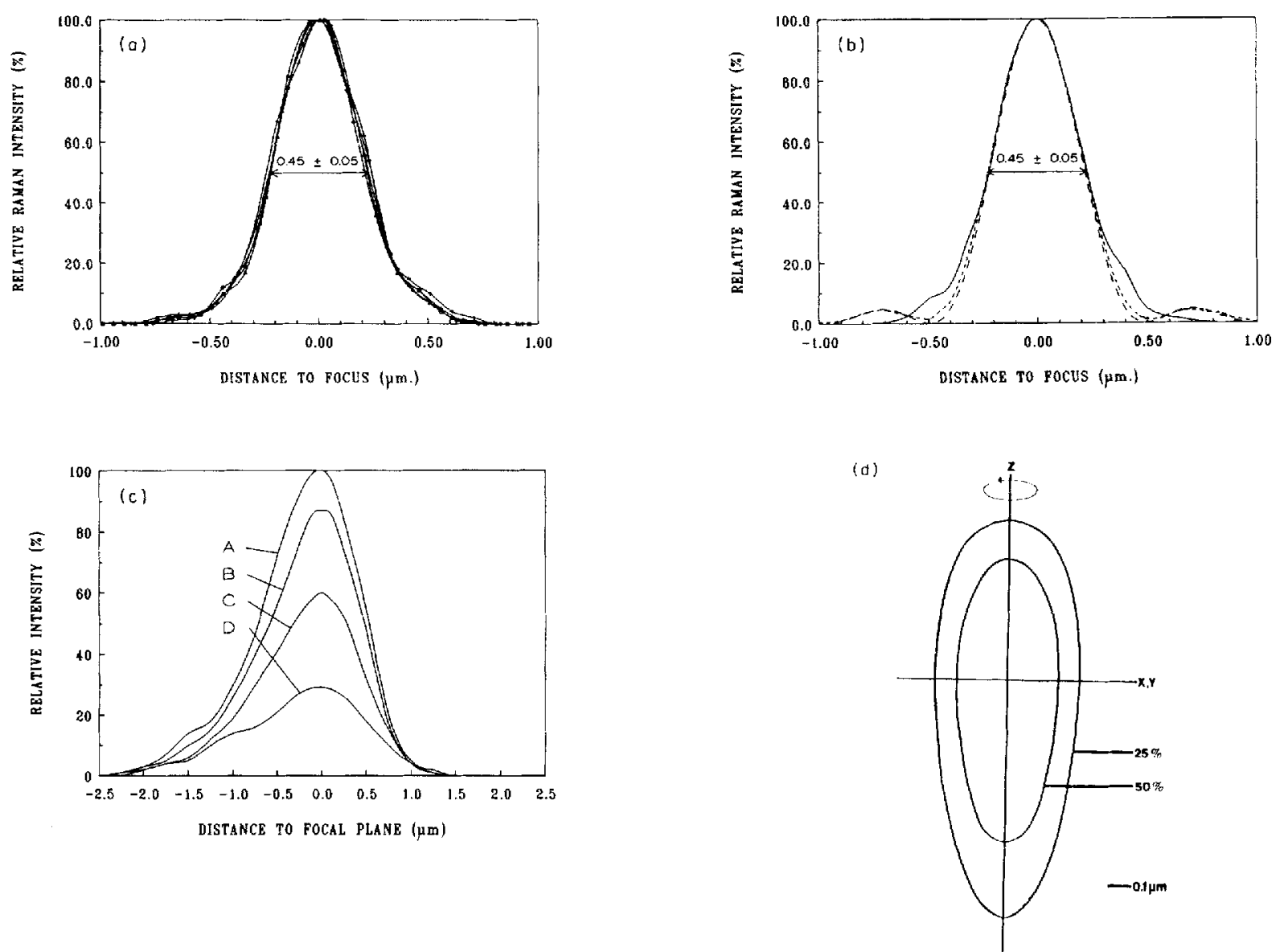

Figure 9. Determination of the three-dimensional measuring volume of the CRM for a $63 \times$ Zeiss Plan Neofluar water immersion objective $(1.2 \mathrm{NA})$ in combination with a pinhole of $50 \mu \mathrm{m}$ radius for $660-\mathrm{nm}$ laser radiation. Results obtained by measuring the intensity of the 1004 $\mathrm{cm}^{-1}$ Raman line of a polystyrene sphere $(0.22 \mu \mathrm{m}$ diameter) as a function of distance to the centre of the laser focus. (a) Results of five scans in the focal plane through the centre of the laser focus, in the direction of the polarization of the laser radiation. (b) As (a), but in the direction orthogonal to the laser radiation polarization (average of five scans, solid line). Also shown are computer simulated response (short dashed line) and $\operatorname{sinc}^{2}$ function (long dashed line). (C) Results of scans in the vertical direction: averages of (A) five scans and (B)-(D) three scans; spread in FWHM values: (A) 0.1, (B) and (C) 0.2, (D) 0.3 . (A) Along the optical axis [as Fig. 7(D)]: (B) $0.1 \mu \mathrm{m}$ off optical-axis; (C) $0.2 \mu \mathrm{m}$ off optical-axis; (D) $0.3 \mu \mathrm{m}$ off optical-axis. (d) Impression of the measuring volume (50\% and $25 \%$ boundaries) based on the results shown in (a), (b) and (c).

experimental curve develops shoulders. The cause of this phenomenon has not been investigated yet (see Discussion). The simulation showed that the broadening of the response curve due to the finite dimensions of the bead is very small $[<0.02 \mu \mathrm{m}$; see Fig. 9(b)]. As this is well within the margin of error of the measurements, the results have not been corrected for this effect. The FWHM in the axial direction is $1.3 \pm 0.1 \mu \mathrm{m}$ [Fig. 7(D)]. Additional measurements were made in which the bead was moved vertically through the focal plane at distances of $0.1,0.2$ and $0.3 \mu \mathrm{m}$ from the optical axis [Fig. 9(c)]. The results of these measurements together give an impression of the dimensions and form of the measuring volume [Fig. 9(d)]. The total volume contained within the FWHM boundaries is about $0.2 \mu \mathrm{m}^{3}$ ( $0.5 \mu^{3}$ for $25 \%$ boundaries).

\section{DISCUSSION}

The potentials and some applications of the CRM in biological studies at the (sub)cellular level have been discussed previously. ${ }^{5}$ The sensitivity and spatial resolution of the CRM are such that Raman spectra can be obtained from virtually any region in a cell or a (metaphase or polytene) chromosome that can be resolved in an optical microscope.

The development of the CRM therefore permits the application of the extensive knowledge, gained from Raman studies of model systems, to the in situ and in vivo characterization of biological (macro)molecules at the single cell level. Figure 10 illustrates the kind of experiments that can now be carried out on a routine basis. It shows spectra obtained from (A) the nucleus and (B) the cytoplasm of an intact human lymphocyte. The lines in the nucleus spectrum can all be assigned to DNA and protein vibrations. DNA lines are absent from the cytoplasmic spectrum, but in this spectrum some phospholipid signal contributions are suspected.

The use of laser radiation of $660 \mathrm{~nm}$ prevents sample degradation $^{22}$ and moreover offers the opportunity to perform Raman measurements on (immuno)fluorescently labelled samples. The absorption band of many fluorescent dyes, such as the commonly used fluorescein isothiocyanate (FITC), is located in the blue or green part of the spectrum, far away from the laser wavelength. The fluorescent label is not excited by the laser 


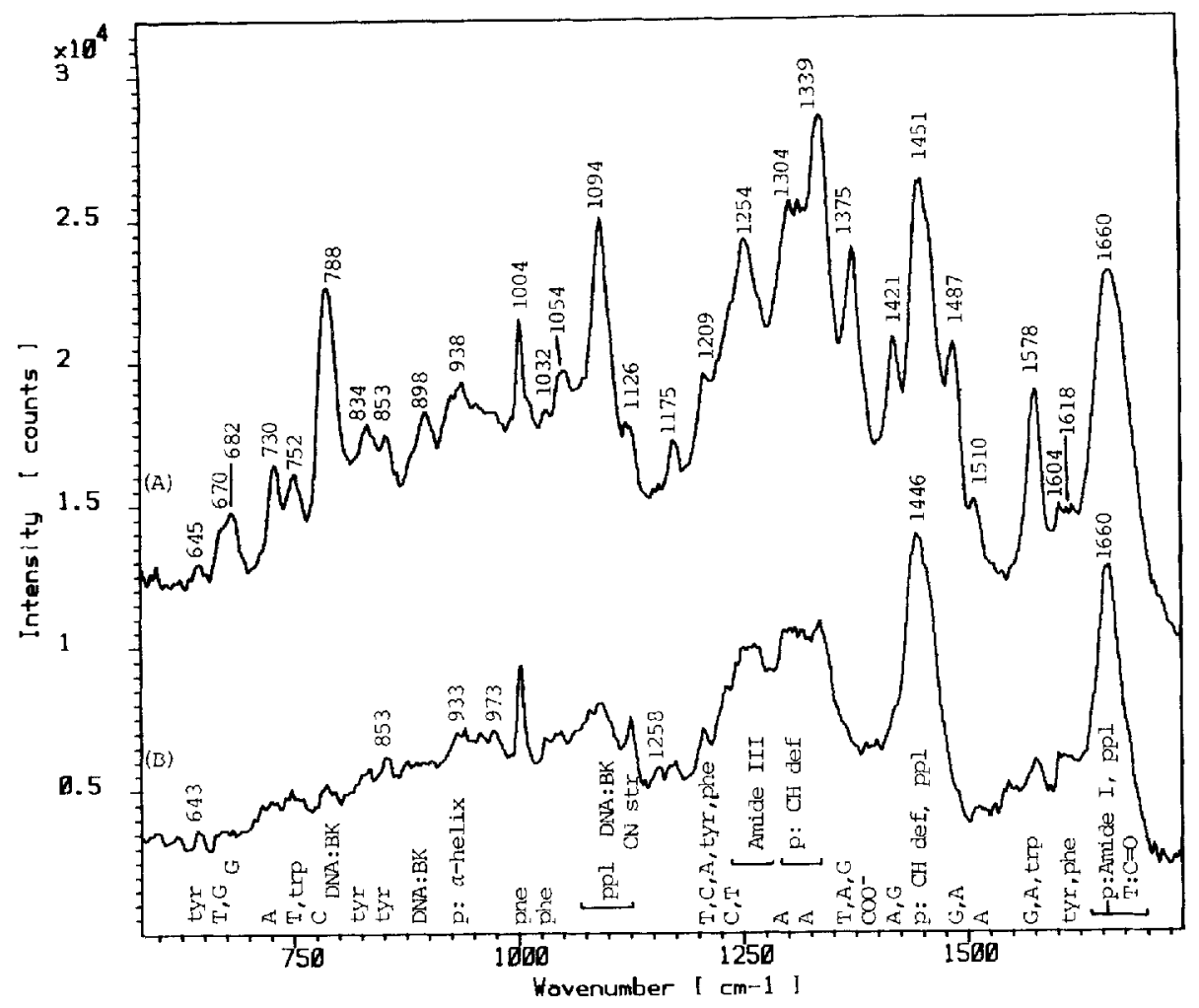

Figure 10. Raman spectra obtained from a single intact human lymphocyte. (A) nucleus (shifted along ordinate for clarity); (B) cytoplasm. Experimental conditions: laser power on the sample, $8 \mathrm{~mW}(660 \mathrm{~nm})$ : microscope objective and pinhole, see Fig. 5; measuring time, $150 \mathrm{~s}$. Raman line assignments based on Refs 23-27. Abbreviations: $T=$ thymine; $C=$ cytosine; $A=$ adenine; $G=$ guanine; $B K=$ backbone; $\mathrm{p}=$ protein $; \alpha=\alpha$-hexlix; tyr = tyrosine; phe = phenylalanine; $\mathrm{ppl}=$ phospholipid; def = deformation; str = stretching. DNA (base) assignments are for spectrum A, phospholipid assignments for spectrum $B$.

radiation and therefore fluorescence does not interfere with the Raman signal. This offers the possibility of localizing, for example, specific (fluorescently labelled) DNA structures in a cell or chromosome by means of fluorescence microscopy prior to starting a Raman measurement or to record spectra of cells of specific white blood cell fractions isolated by means of fluorescenceactivated cell sorting on a flow cytometer, after labelling with FITC conjugated antibodies.

Comparison of the experimentally determined spatial resolution of the CRM with reported theoretical work on confocal systems ${ }^{19}$ indicates that (if needed) some improvements should be possible (without changing the pinhole diameter). From published calculations ${ }^{19}$ it was deduced that the theoretically obtainable FWHM values of the point response curves for the present system are $0.31 \times 0.31 \times 0.8 \mu \mathrm{m}^{3}(X \times Y \times Z$, for circularly polarized light). The cause of the difference between the experimental and calculated values has not been extensively investigated yet. The asymmetry of the depth-response curve (Fig. 7) and the shoulders on the radial response curves [Figs 9(a) and (b)] could indicate the presence of some aberrations in the microscope objective. Residual spherical aberration has been found to be present in many high numerical aperture objectives. ${ }^{21}$ This could be aggravated in the present situation because the objective is employed at higher wavelengths than is usual in conventional microscopy and therefore outside the wavelength region for which aberrations are optimally corrected.

Another possibility is that the numerical aperture $(N A)$ of the objective is less than 1.2 in the wavelength region where the CRM is operated $(660-850 \mathrm{~nm})$. However, to explain the experimental results, the $N A$ would have to be 0.8 instead of 1.2 , which is unlikely. The beam quality of the dye laser may not be optimum, which will also affect the quality of the laser focus. This could be improved by spatial filtering of the laser beam.

The fact that the spatial resolution is not close to its theoretical limit may also explain why for $X$ (perpendicular to laser radiation polarization) and $Y$ (parallel to laser radiation polarization) resolution the same value was found, whereas theory predicts a larger FWHM value for the $X$ - than for the $Y$-direction. ${ }^{19}$

Further substantial improvements of the CRM regarding sensitivity will have to come from detectors with an even higher quantum efficiency or from a higher signal collection angle. Regarding the first point it should be noted that back-thinned CCD chips do not have a (much) higher quantum efficiency than normal chips at wavelengths $>700-750 \mathrm{~nm}$. Chips with a higher number of pixels (which are now commercially available) would permit the recording of a wider spectral interval (or can be used to reduce aliasing effects). For very thin samples, e.g. Langmuir-Blodgett films, the solid angle for signal collection can simply be doubled by placing the sample on a mirror. A test (not shown) with a metaphase chromosome (chromatid diameter about $0.5-1 \mu \mathrm{m}$ ) on a dichroic mirror (reflecting Raman scattered light and transmitting light of $<680 \mathrm{~nm}$, thus allowing the use of the illumination system in the base of the microscope frame for localization of the chromosome) led to an average increase in Raman intensity from chromosomes of $25 \%$ and, equally 
important, blocked signal contributions from the fused silica.

Another possibility for further improvement is to take full advantage of the fact that a CCD camera is a twodimensional detector. This can be realized by line illumination of the sample instead of spot illumination, as was described by Bowden et al. ${ }^{28}$ The pinhole then has to be replaced by a slit, but the depth resolution will suffer. This can be compensated for by reducing the slit width at the cost of a lower signal intensity. Line illumination would make it possible to record Raman spectra from different parts of a sample simultaneously. Not only would it facilitate the study of, for example, chromosomal banding patterns, but it could also yield important local information in situations where changes in cells take place, e.g. in cell-cell or cell-drug interactions.

Finally, combining line illumination and automated scanning of the sample in the two remaining dimensions, three-dimensional imaging of cells on the basis of the intensity of a selected Raman line should become possible.

\section{Acknowledgements}

The authors thank the Dutch Foundation for Biophysics for financial support and B. J. M. Leerkotte, H. A. de Boer and H. W. Krabbe for expert technical assistance.

\section{REFERENCES}

1. For reviews see, e.g. R. J. H. Clark and R. E. Hester (Eds). Advances in Spectroscopy, Vol 13, Spectroscopy of Biological Systems. Heyden, London (1986).

2. W. L. Kubasek, Y. Wang, G. A. Thomas, T. W. Patapoff, K.-H. Schoenwaelder, J. H. van der Sande and W. L. Peticolas, Biochemistry 25, 7440 (1986).

3. F. Sureau, L. Chinsky, C. Amirand, J. P. Ballini, M. Duquesne, A. Laigle, P. Y. Turpin and P. Vigny, Appl. Spectrosc. 44, 1047 (1990).

4. B. Baek, W. H. Nelson, D. Britt and J. F. Sperry, Appl. Spectrosc. 42, 1312 (1988)

5. G. J. Puppels, F. F. M. de Mul, C. Otto, J. Greve, M. RobertNicoud, D. J. Arndt-Jovin and T. Jovin, Nature (London), 347, 301 (1990).

6. L. W. M. M. Terstappen, B. G. de Grooth, G. M. J. Nolten, C. H. H. ten Napel, W. van Berkel and J. Greve, Cytometry 7, 178 (1986).

7. G. J. Rosasco, E. S. Etz and W. A. Cassatt, Appl. Spectrose. 29,396 (1975)

8. M. Delhaye and P. Dhamelincourt, J. Raman Spectrosc. 3, 33 (1975).

9. G. J. Puppels, A. Huizinga, H. W. Krabbe, H. A. de Boer, G. Gijsbers and F. F. M. de Mul, Rev. Sci. Instrum. 61, 3709 (1990).

10. CCD Imaging III. English Electric Valve, Chelmsford, Essex (1987).

11. R. Howard and M. Maynard, App/. Spectrosc. 40, 1245 (1986).

12. G. J. Brakenhoff, P. Blom and P. Barends, J. Microse. 117, 219 (1979).
13. I. J. Cox, C. J. R. Sheppard and T. Wilson, Optik 60, 391 (1982).

14. J. G. White, W. B. Amos and M. Fordham, J. Cell Biol. 105 , 41 (1987).

15. K. Carlsson and N. Åslund, Appl. Opt. 26, 3232 (1987).

16. T. Wilson and A. R. Carlini, J. Microsc. 149, 51 (1988).

17. T. Wilson and A. R. Carlini, Opt. Lett. 12, 227 (1987).

18. T. R. Corle, C.-H. Chou and G. S. Kino, Opt. Lett. 11, 770 (1986)

19. H. T. M. Van der Voort and G. J. Brakenhoff, J. Microsc. 158 , $43(1990)$.

20. E. S. Etz and J. J. Blaha, Natl Bur. Stand. (U.S.) Spec. Publ. No. $533,153(1980)$

21. Y. Hiraoka, J. W. Sedat and D. A. Agard, Biophys. J. 57, 325 (1990).

22. G. J. Puppels, J. H. F. Olminkhof, G. J. M. Segers-Nolten, C. Otto, F. F. M. de Mul and J. Greve, in preparation.

23. G. J. Thomas, Jr, B. Prescott and D. E. Olins, Science 197, 385 (1977).

24. R. Savoie, J.-J. Jutier. S. Alex, P. Nadeau and P. N. Lewis, Biophys. J. 47, 451 (1985).

25. C. Otto, PhD Thesis, University of Twente, Enschede (1987),

26. A. T. Tu, in Advances in Spectroscopy, Vol. 13, Spectroscopy of Biological Systems, edited by R. J. H. Clark and R. E. Hester, pp. 47-112. Heyden, London (1986).

27. A. T. Tu, Raman Spectroscopy in Biology, Principles and Applications. Wiley, New York (1982).

28. M. Bowden, D. J. Gardiner, G. Rice and D. L. Gerrard, J. Raman Spectrosc. 21, 37 (1990). 